\title{
Echocardiographic prediction of surgical reparability in degenerative mitral regurgitation due to leaflet prolapse: a review
}

\author{
Francesca Mantovani, Francesca Bursi, Giovanna Di Giannuario \& Andrea \\ Barbieri
}

To cite this article: Francesca Mantovani, Francesca Bursi, Giovanna Di Giannuario \& Andrea Barbieri (2019): Echocardiographic prediction of surgical reparability in degenerative mitral regurgitation due to leaflet prolapse: a review, Expert Review of Cardiovascular Therapy, DOI: 10.1080/14779072.2019.1664289

To link to this article: https://doi.org/10.1080/14779072.2019.1664289

Accepted author version posted online: 04

Sep 2019.

Submit your article to this journal

Q View related articles $\asymp$

View Crossmark data 
Publisher: Taylor \& Francis

Journal: Expert Review of Cardiovascular Therapy

DOI: $10.1080 / 14779072.2019 .1664289$

\section{Review}

Echocardiographic prediction of surgical reparability in degenerative mitral regurgitation due to leaflet prolapse: a review

Francesca Mantovani ${ }^{1}$, Francesca Bursi ${ }^{2}$, Giovanna Di Giannuario ${ }^{3}$ \& Andrea Barbieri ${ }^{4}$

1. Department of Cardiology, Azienda Unità Sanitaria Locale - IRCCS di Reggio Emilia, Italy

2. Department of Cardiology, Azienda Socio Sanitaria Territoriale (A.S.S.T.) SANTI PAOLO E CARLO - Presidio Ospedale San Paolo, Milano, Italy

3. Department of Cardiology, Ospedale "Infermi" di Rimini - AUSL Romagna, Italy

4. Department of Cardiology, Azienda Ospedaliera-Universitaria di Modena, Italy olmoberg@libero.it

\section{Corresponding author:}

\section{Andrea Barbieri}

Department of Cardiology, Azienda Ospedaliera-Universitaria di Modena, Modena, Italy Email: olmoberg@libero.it 


\begin{abstract}
Introduction. Despite current guidelines provide recommendations for the optimal management of degenerative mitral regurgitation (MR), this condition remains often undertreated with delay in surgical referral and dismal effect on outcomes.
\end{abstract}

Areas covered. This review focuses on the role of echocardiography in guiding mitral valve (MV) surgical repair in degenerative MR due to leaflet prolapse.

Expert opinion. A stepwise protocol-driven echocardiography shared by referring physician and surgeon may help to guide referral to surgical repair in degenerative MR. This protocol particularly is useful to identify the ideal patho-anatomy for a successful and durable repair especially when early surgery is proposed and to refer the patient to centers of excellence in case of complex anatomy. Nearly $100 \%$ repair rate can be achieved when the surgical technique is adapted to the lesions seen in each valve. Threedimensional echocardiography predicts repair complexity may be useful and should therefore be implemented. However, the current literature is far from comprehensive deriving from small, single-center studies. Therefore, reproducibility and external validation, especially with newly developed quantitative automated software, are still needed.

\title{
Keywords
}

Degenerative mitral regurgitation; Mitral Valve Prolapse; Echocardiography; Surgery; Repair 


\section{Article highlights box}

- Despite current guidelines on management of degenerative mitral regurgitation (MR), this condition remains often undertreated with delay in surgical referral and dismal effect on outcomes.

- A stepwise protocol-driven echocardiography shared by referring physician and surgeon may help to guide referral to surgical repair in degenerative MR. This protocol particularly is useful to identify the ideal pathoanatomy for a successful and durable repair especially when early surgery is proposed and to refer the patient to centers of excellence in case of complex anatomy.

- A systematic step by step approach is recommended since a combination of lesions may coexist and only the correction of all valve dysfunctions will allow a successful repair. The first step is to identify and characterize the main (principal) lesion, then to quantify it and finally identify potential secondary mechanisms that could be masked by the obvious lesion.

- Although it has been shown that three-dimensional echocardiography accurately predicts repair complexity, there are no randomized trial examining the role of this technique in guiding indication to surgery.

- A retrospective study based on comparison of historical data showed that defined protocol-driven preoperative echocardiography improved repair rate and intraoperative concordance between imaging expert and surgeon.

- Maximized repair rate strategy can be achieved when the surgeon adapts various surgical techniques to the lesions seen in each valve. Therefore, the repair technique chosen by the surgeon requires more input from the three-dimensional echocardiography study.

\section{Introduction}

Degenerative mitral regurgitation (MR) represents an important disease given its prevalence, adverse prognostic impact, and symptoms burden associated with progressive left-sided heart failure.[1, 2, 3, 4] Population level data showed that these patients have decreased survival with mortality rates projected to double over the next 25 years. [5]

The revised European Society of Cardiology and American Heart Association/American College of Cardiology guidelines provide recommendations for the optimal management of MR. [6, 7] However, knowledge gaps for patients undergoing surgical repair [8, 9] impede their implementation. [10] Specifically, adherence to guidelines suggesting early referral for mitral valve (MV) repair in order to avoid permanent damage on left ventricle is low, in part because the rate of surgical repair is considered to be too low in some centers. As a result, degenerative MR is often undertreated with delay in surgical referral, causing overuse of medical therapy,[11] higher operative risk and potential irreversible sequelae even in community with state-of-the-art facilities, good access to treatment, and high availability of specialized providers.[12] 
In this context, echocardiographic focused educational and practice interventions should be developed in order to implement access to high-quality MV interventions. [13] Accordingly, consistent anatomical and functional descriptors of MV lesions can be crucial to assess surgical reparability and to guide surgical techniques and consequently improve outcomes, since it is now proven that surgical correction is associated with long-term improved survival and low heart failure risk regardless of patients' age, [14] if performed before irrecoverable left heart dilation occurs, in valve centers offering low operative risk. [15, 16]

However, current data from the Society of Thoracic Surgeons database indicate a MV repair rate well below 90\% for degenerative disease among 867 centers in North America.[17]

Hence, this review focuses on the added value of echocardiographic-guided MV repair in order to influences the rate of successful MV repair potentially preventing excess mortality and morbidity related to degenerative MR by assisting clinicians in defining the right surgeon-valve match (more advanced MV prolapse should best be operated by a reference $\mathrm{MV}$ surgeon) and the adequate and personalized timing strategy in patients with degenerative MR due to leaflet prolapse.

\section{Improve MV repair rates by protocol-driven echo guidance}

Theoretically, standardized protocol-driven preoperative echocardiography could serve as a basis for selective referral through the improvement of intraoperative concordance. A well-performed transthoracic two-dimensional (2D) echocardiogram allows to outline most of the mechanisms of MV disease (Figure 1). [18] However, recent advances in transesophageal three-dimensional echocardiography (3D-TEE) consent clearer identification of causative etiology of MR and may help to define surgical planning before entering the operating room. [19].

Three-dimensional TEE revolutionized the ability to visualize MV anatomy in vivo with definitive advantages over flaccid heart examination made by surgeons. Compared to 2D-TEE, 3D-TEE is less operator dependent because the entire MV can be visualized in a single view, consenting simultaneous examination of both leaflets from either the left ventricular or the left atrial perspective[20].

The incremental value of 3D-TEE seems especially important in the subset of patients with complex multisegment MV disease. [21]

It is therefore of increasing importance that the contemporary MV surgeon possesses an in-depth knowledge of MV echocardiographic imaging and interpretation. Likewise, it is equally important that referring cardiologists understand the various surgical techniques available and how to provide echocardiographic views of the MV to optimally aid in planning surgery.

For the referring physician, the protocol-driven echo guidance basically has two objectives: 1) identify the ideal pathoanatomy for effective surgical repair, especially in asymptomatic patients without class Ila indications for surgery according to the American Heart Association/American College of Cardiology 
guidelines (early surgery) [22], 2) identify patients with complex repairs to refer to centers of excellence in a timely fashion.

\section{Step-wise protocol-driven echo guidance categorizes the spectrum of degenerative mitral regurgitation}

Mitral regurgitation is a conundrum in terms of mechanism. Consequently, a systematic step by step approach is recommended since a combination of lesions may coexist and only the correction of all valve dysfunctions will allow a successful repair.

\subsection{Step-1: focus on identifying the main (principal) lesion}

The pathoanatomic approach represent the crucial strategic roadmap toward successful repair (Figure 2) [23].

In degenerative pathologies, MV repair is most likely in patients with MR due to excessive leaflet motion (Type II) and is least likely in MR due to restricted leaflet motion both in systole and diastole (Type IIIa). [24] Of note, patients with Type II disease may have combined pathologies such as focal restrictive Type IIIA or IIIB disease. One should be particularly suspicious of this when patients with degenerative MR present with left ventricular dilatation. Patients with true mixed etiologies deserve special attention as this may increase the technical complexity to achieve a durable repair (Video 1 ).

\subsection{Step-2: quantify the main (principal) lesion}

Once the principal lesion has been identified, it is important to ascertain also the tissue features of the leaflets involved in the pathology. In general, extensive chordal or leaflet disruption or the presence of mitral annular calcification reduce the feasibility and likelihood of successful MV repair. Differentiation of the degenerative etiology into different phenotypes, the diffuse degenerative disease (also named as Barlow's disease) and fibroelastic deficiency, is the key factor to stratifying complexity, but the spectrum of pathology varies widely, such that many valves cannot be so simply categorized and can be considered "forme fruste". [25]

Fibroelastic deficiency is a focal fibrillin deficiency, most commonly affecting the medial posterior (P2) scallop and associated with overall thin leaflet tissue and focally ruptured degenerative chordae. [26] This often-focal pathology affords a focal surgical solution. The diffuse degenerative disease includes excess leaflet tissue secondary to mucopolysaccharide accumulation in leaflet mucosa and extensively involves both leaflets with multi-segmental prolapse.

Of note, quantitative analysis of MV anatomy from 3D-TEE data has been shown to clearly differentiate diffuse degenerative disease from fibroelastic deficiency disease. [27] The surgical approach to each level of this spectrum is different and pathoanatomically directed (Table 1). The probability of MV repair is most likely with posterior prolapse or flail, whereas isolated anterior leaflet or bileaflet prolapse is more difficult to repair (Video 2,3). However, P2 prolapse is not always so simple if 
accompanied by mitral annular calcium or hypoplasia of the opposite leaflet and extreme fibroelastic deficiency. [28]

In organic MR, some quantitative and semiquantitative predictors of unsuccessful repair have been reported: extensive prolapse ( $\geq 3$ scallops especially if the anterior leaflet is involved, lateral commissure, extensive calcification, annular dilatation $>50 \mathrm{~mm}$ ). By convention, the likelihood of repair could be generically classified into either "feasible", "difficult", "unlikely" [29] or "ideal", "challenging" and "relatively contraindicated"[30]

Nevertheless, a classification of unlikely/relatively contraindicated would not mean impossible but this would require greater surgical judgement and a higher degree of technical proficiency.

3.3 Step-3: identify potential secondary mechanisms that could be masked by the main lesion

As the degree of myxomatous degenerative involvement increases, so does the potential existence of these secondary mechanisms. It is very important to identify them since resection or other reconstruction will change the valve closing dynamics and may make other valve lesions more prominent which may lead to residual MR.

Three-dimensional echocardiography is the essential step to refine the pathoanatomy of the lesion(s) (Figure 3).[21] The examination should not focus on the main lesion such as a P2 flail, but on identifying the potential secondary mechanisms that could be masked by a main dominant lesion. After a thorough 2D-TEE assessment, where the majority of these features can be identified, [31] 3D-TEE has the diagnostic sensitivity to highlight potential subtle surgical pitfalls such as the coexistence of anterior mitral leaflet prolapse masked by an main posterior mitral leaflet lesion (Video 4), the presence of commissural prolapse at the medial (Video 5) or lateral location as a separate lesion (Video 6), posterior mitral leaflet clefts/indentation adjacent to the primary lesion (Video 7) or anterior mitral leaflet secondary chordal tethering resulting in lack of coaptation [32] and predictors of systolic anterior leaflet motion (coaptationseptal distance $<25 \mathrm{~mm}$, sharp aorto-mitral angle $<120^{\circ}$, posterior mitral leaflet height $>15 \mathrm{~mm}$, small left ventricular end-diastolic cavity diameter $<45 \mathrm{~mm}$, nonenlarged annulus, basal septal thickness $>15 \mathrm{~mm}$ ) (Figure 4, Video 8). [33] Therefore, avoiding under sizing of the annuloplasty ring is very important in patients with these risk factors.

Cross-plane of the 3D-TEE surgical view makes easier to measure the prolapse height of every segments (Figure 5).[34]

Importantly, during surgical inspection, while the heart is flaccid, the clefts and indentations are difficult to identify.[35] Similarly, the identification of commissural lesions has greatly improved with 3D-TEE with published sensitivity, specificity and accuracy ranging from $89 \%$ to $92 \%, 95 \%$ to $99 \%$ and $93 \%$ to $97 \%$, respectively[21]. 
A current limitation of the echocardiographic examination is that little attention has been paid so far to the impact of annular dynamics on mitral regurgitation mechanisms. Indeed, MV pathoanatomy cannot be confined to leaflet pathology, since degenerative MV disease involves the entire mitral apparatus (the valvulo-ventricular complex), included mitral annulus, as confirmed by the recognized role of annuloplasty in ensuring successful MV repair [36]. The advent of 3D-TEE allowed to highlight that annular disfunction with the saddle shaped loss of the MV annulus is a frequent finding in degenerative MV disease[19], especially in diffuse degenerative phenotype, compared to relatively preserved annular function in fibroelastic deficiency[37]. Future research directions will require to sought whether these differences in annular dynamics can be addressed differently by surgical annular devices and techniques. The growing importance of evaluating all the components of the valvulo-ventricular complex to correctly plan MV repair before entering the operating room will require to develop a standardized set of dynamic measurements in the future, included the quantitative degree of annular enlargement and dysfunction, the valvular leaflets' redundancy or paucity, the prolapse characteristics (volume and height). For now, current measurements of 3D-TEE dynamic characteristics of the mitral valvulo-ventricular complex are time consuming and are unlikely to enter clinical practice if not rendered at least semiautomatic in the future.

Another limitation of echocardiography is that in some patients, specific MV image features are not optimally displayed by conventional 3D-TEE rendering. Transillumination is a new 3D rendering tool that uses a freely movable virtual light to enhance image details and depth. In a preliminary report it appears to increase the diagnostic confidence of the readers when it is used to improve delineation of orifices, borders, cavities and structural abnormalities [38].

\section{Echocardiographic guidance in order to increase surgical repair rate: what is the evidence?}

It is fair to remember that there is a small subgroup of patients with degenerative MR in whom valve replacement may still be preferred over valve repair, such as those who have had a prior cardiac operation or prior chest radiation, in whom any subsequent operation for a failed repair would be undertaken at substantially increased risk and percutaneous treatment is not feasible. [30]

Although it has been shown that 3D-TEE accurately predicts repair complexity [39], its clinical downstream effects are challenging to investigate. Indeed, the evidence-based comparison of MV repair with unguided and echo-guided procedures is difficult because restricting the surgeon's access to echocardiographic information or prolonging intraoperative cardiac arrest for blinded evaluation poses ethical issues. Therefore, an observational rather than randomized design with historical institutional and/or concomitant national results used as controls is the only feasible study design. Moreover, the skills and experience of the surgeons in the specialty of MV repair are the most important issues that affect repair rate and its 
durability. [40] Still, no controlled study will ever provide this intangible evidence because valvular surgery in this context is a very "surgeon and center-dependent" therapy as the ability to perform several techniques [41]. On the other hand, too much credence on echocardiography may be of concern and clinical evidence on the incremental benefit of 3D-TEE is needed. In fact, the echocardiography-based approach could sometimes be misleading: a lesion echocardiographically interpreted as simple could be sent for repair to a low-volume center, where unexpected surgical finding could end with a replacement of MV. [39]

For all these reasons, the efficacy of echo-guided mitral repair had not been formally established until 2014, when Drake et al. demonstrated that a defined protocol-driven preoperative echocardiography improved intraoperative concordance and repair rate.[42] From July 1, 2000, through June 30, 2010, 347 consecutive adult patients with MV disease were referred for surgery. Among those with surgical indication, in the first 101 unguided controls the decision to attempt repair was based on direct mitral evaluation in the arrested heart. Subsequently, 237 underwent protocol-driven echo-guided intervention. Repair was attempted in $81.0 \%$ of the echo-guided group compared with $51.5 \%$ of unguided controls $(p<0.001)$. Of note, under echo-guidance, $99 \%$ of the 237 patients and their referring physicians were explicitly informed of the intent to repair or replace well in advance of surgery. In the 45 patients who received replacement, $93 \%$ were preoperatively informed that repair was unlikely or would not be attempted.

\section{Perception of mitral valve repair complexity differs between operators}

Although complex MV lesions have been recognized as being in general more difficult to repair, actually no precise description is available to prospectively identify the lesions' complexity. Indeed, a literature review did not yield any studies investigating the complexity of valve repair with regression analysis that could have assigned objective weightings for the specific MV lesions. Therefore, in clinical practice, it is used an arbitrary approach. To try to overcome these limitations, Anyanwu et al. [43] proposed a complexity scale using a consensus approach, assigning a score to each valve derived from intraoperative observation (Figure 6).

A technique score was also calculated by adding the number of principal techniques used to repair each valve. Of note, in $51 \%$ of complex cases (compared with $14 \%$ of intermediate cases, and none of the simple cases) $>1$ advanced surgical technique was necessary to execute repairs.

This approach is attractive for the referring cardiologist since all the components of the score may be evaluated by preoperative echocardiography. However, while this study can be considered a first step toward a more general classification of lesions and surgical techniques that could be universally applied, no external and prospective validation was done. Indeed, for any given particular MV lesion, several surgeons might use multiple repair maneuvers, making it a complex repair, whereas others might use a simpler 
approach. On the other hand, the effort for an effective standardization is necessary not solely to allow to achieve higher global repair rates, but to make cardiologists feel more comfortable in referring to surgery also patients with complex lesions earlier in the course of the disease, reducing left ventricular permanent damages.

\section{Echo-guided in mitral "repair-all" strategy}

The evolution of $M V$ surgical repair techniques in to the current era have led to better outcome of repair for all subsets. Indeed, the durability of anterior leaflet repair is currently statistically indistinguishable from repair of other leaflet subsets and mechanical valve replacement.[44]

This information suggests that outcome of correction of anterior or bileaflet disease approaches that of posterior leaflet repair and do not support the perception by many clinicians that results of valve replacement for mitral prolapse are more predictable as regards subsequent risk of reoperation. Nevertheless, a recent joint report of the American Association for Thoracic Surgery, American College of Cardiology, American Society of Echocardiography, Society for Cardiovascular Angiography and Interventions, and Society of Thoracic Surgeons proposed that a designated valve center should exceed that average and achieve a surgical MV repair rate of greater than $75 \%$ for patients with degenerative MR in the absence of calcification of the leaflets or annulus. [45] Indeed, according to this criterion, up to $25 \%$ of patients may require MV replacement. Therefore, many young patients may receive a mechanical MV, with the associated significant increase risk of bleeding, ischemic stroke and death.[46] This gave the impetus to evolve the concept of high-volume centers of excellence in MV repair. Accordingly, recent reports showed very high rates of $\mathrm{MV}$ repair associated with low mortality in all types of valve complexity and age groups.[47]

The nearly $100 \%$ repair rate strategy can be achieved when the surgeon adapts various surgical techniques to the lesions seen in each valve. Therefore, the repair technique chosen by the surgeon requires more input from the 3D-TEE study. [39] In high volume center, intra- operative echo-guided revision of the initial repair is performed in most patients without a significant incremental risk.[48] However, in general practice, anterior or bileaflet prolapse continue to be significantly more difficult to repair than isolated posterior leaflet prolapse [49] with less long-term durability. [50]

\section{What are the difficulties that hinder the echo-guided mitral repair strategy?}

Many would consider MV repair to constitute a surgical subspecialty. [51] [52] Today, no more than 5-10\% of patients should undergo an 'unanticipated replacement' or have more than mild residual regurgitation if operated on in a 'reference center'.[53]

Although some pathologic conditions may be repaired with a high degree of certainty by experienced surgeons, considerable variation still exists. A recent large statewide clinical registry showed that between 
patients with asymptomatic chronic severe degenerative MR and preserved left ventricular function, one in five patient underwent replacement.[54] This shows the importance of regionalizing MV surgery in centers of excellence at a time when the current European Society of Cardiology and American Heart Association/American College of Cardiology guideline recommend to intervene earlier in patients with severe MR on the basis of degenerative valve disease.[6] [7] However, it is noteworthy that there is considerable practice pattern variation among surgeons in the choice of techniques to use, with an inverse relationship between institutional and surgeon volume and rate of mitral repair (rather than replacement) after adjustment for pre-operative risk factors. Interestingly, data from New York State suggest that lowvolume surgeons ( $<25$ operations/year) operating in institutions where high-volume and high-repair-rate surgeons are present ( $>50$ operations/year and $>70 \%$ repair rate) have improved their repair rates, underlying the importance of the setting for personal improvement of surgical skills.[55] Using this observational study as a single example, an appropriate cut-off for low and high surgical volumes for mitral valve operation might be $>25$ per year per operator or $>50$ per year per institution. [45] However, further observational studies are needed to validate these cut-offs. Indeed, it must also be noted that while volume is an important component of surgical outcome, the case-mix complexity must also be high to maintain high competency. [39]

Yet, not just as the surgeon should have a strong focus on mitral repair but also the cardiology colleagues. Indeed, by combining patient evaluations with appropriate utilization of echocardiography, practice-level clinicians play a vital gatekeeper role within a system of care for degenerative MR but several echo laboratories report the presence and severity of MR but not the pathoanatomic mechanism. $[11,56]$ Consequently, when significant MR is suspected or confirmed, most patients should be referred to a local or regional cardiovascular specialist for further evaluation and management[45] but too many patients are not (probably ever) presented in centers with adequate expertise for echocardiographic evaluation. As a result, still too many patients are allocated to treatment strategy solely depending on the «experience» of the treating physician, which is often watchful-waiting on the basis of the "primum non nocere» principle (the so-called "invisible patients" in mainstream clinical practice). [57] For this reason, defined pathways for patient referral that incorporate bidirectional communication between primary and subspecialty providers should be created. As the extent of diagnostic approaches to degenerative MV continues to expand and as surgical treatment options continue to evolve, educational programs directed at the primary care provider assume increasing importance [11].

\section{Transcatheter mitral valve chord repair: the new surgical approach}

The MV repair with implantation of polytetrafluoroethylene neochordae using transapical catheter-based techniques represents an appealing treatment of degenerative $M R$, as the correction of $M V$ prolapse is performed in beating heart under full left ventricular loading conditions and echocardiographic guidance 
through a small incision. Therefore, this concept enables a physiologic approach to a complex problem based on a solid surgical background.

NeoChord was the first device to obtained the CE Mark in 2012 after the initial results that reported an overall reduction of MR to $\leq 2+$ in $86.7 \%$ among 30 patients in the safety feasibility Transapical Artificial Chordae Tendinae (TACT) trial[58].

In select patients with isolated posterior leaflet prolapse of the $\mathrm{P} 2$ or $\mathrm{P} 3$ segment, or both, MV repair using the NeoChord system results in very good long-term results without recurrent prolapse, MR, or annular dilatation[59].

So far, NeoChord DS 1000 device has been used in nearly 1,000 patients, even if a relative high number of implants are required to overcome the learning curve[60].

Recently, a multicenter study conducted in Europe showed excellent results with the reduction of MR to mild or less residual in $96.7 \%$ of patients who received $\geq 2$ neochordae. At 1-year follow-up, $98 \%$ survival and $84 \%$ freedom from major cardiac adverse events were observed. However, the complexity of the lesion is clearly affecting the reduction of MR[61].

Although preliminary data reveal that the lack of an annuloplasty ring does not lead to ongoing annular dilatation and does not induce recurrent MR, it is conceivable that intervention before left ventricular remodeling may be particularly important for durability of the Neo-Chord procedure because reverse remodeling may result in shortening of the distance from the repaired MV leaflet to the insertion point on the left ventricular wall, thus possibly resulting in recurrent MV prolapse and MR.

Currently, patients with isolated posterior leaflet prolapse, in particular P2, and only limited annular dilatation seem to be the best target population for this procedure. However, the "transcatheter MV chord repair concept" have the potential to simplify and increase the quality and rates of MV repair and decrease the morbidity associated with conventional open cardiac mitral operations.

Certainly, further investigation with longer-term follow-up and direct comparison with conventional MV surgery is necessary to validate this concept. The Randomized Trial of the NeoChord DS1000 System Versus Open Surgical Repair (ReChord); (ClinicalTrials.gov Identifier: NCT02803957) is underway. Nevertheless, it is easy to predict that more advances from further development of interventional techniques, better patient selection and intraoperative imaging guidance are expected to advance in the near future. If these presumptions will be confirmed, this less invasive and physiological approach may be used in the very early disease process of degenerative MR in a broader set of patients and may represent an alternative to increase the overall MV repair rate because of its relative technical simplicity. 


\section{Conclusions}

Considering the variety of lesions, surgical repair in degenerative MR due to leaflet prolapse is still an art and has its own set of unique problems. Therefore, it is essential that the surgeons understand more than the basics of MV repair to achieve a good performance because advanced techniques may be required unexpectedly. In contrast, preoperative protocol-driven echocardiography can identify patients with complex pathology that will pose a challenging repair and provide views of the valve to optimally aid in planning therapy and guide intervention. For this reason, the traditional paradigm of reparability based solely on surgical exploration is shifting towards a new standard centered on shared contributions from both imaging expert cardiologists and surgery. Protocol-driven echo guidance, performed prior to surgery, gives the patient, family, and referring physicians ample time to prepare to the proposed intervention and to allocate the patient to the most suitable surgical center.

\section{Expert opinion}

In degenerative MR due to leaflet prolapse, echo-guided repair strategy is crucial in determining whether a patient should be directed to a reference surgeon and has implications in terms of timing of surgery and long-term morbidity and mortality. Although cardiologists agree that early surgical referral of patients with simple MV disease (posterior prolapse) is supported by evidence, many have been reluctant to recommend surgical intervention for asymptomatic patients with complex disease (i.e., anterior or bileaflet prolapse), fearing that surgical outcomes are inferior to watchful waiting. Ultimately, the repair technique chosen by the surgeon requires more input from the 3D-TEE study because it has been shown that a nearly $100 \%$ repair rate can be achieved when the surgeon adapts surgical techniques to the lesions seen in each valve. For these reasons, a multidisciplinary approach is strongly encouraged. One limitation of the current use of $3 D-T E E$ is the lack of large trials comparing the additive value of $3 \mathrm{D}$ over $2 \mathrm{D}$ imaging. Although this information exists in small, single-center studies, reproducibility and validation, especially with newly developed automated quantitative software, are still needed. 


\section{Funding}

This paper was not funded.

\section{Declaration of interest}

The authors have no relevant affiliations or financial involvement with any organization or entity with a financial interest in or financial conflict with the subject matter or materials discussed in the manuscript. This includes employment, consultancies, honoraria, stock ownership or options, expert testimony, grants or patents received or pending, or royalties.

\section{Reviewer Disclosures}

Peer reviewers on this manuscript have no relevant financial or other relationships to disclose

\section{References}

1. Nkomo VT, Gardin JM, Skelton TN, et al. Burden of valvular heart diseases: a population-based study. Lancet. 2006 Sep 16;368(9540):1005-11. doi: 10.1016/S0140-6736(06)69208-8. PubMed PMID: 16980116.

2. Ling LH, Enriquez-Sarano M, Seward JB, et al. Clinical outcome of mitral regurgitation due to flail leaflet. N Engl J Med. 1996 Nov 7;335(19):1417-23. doi: 10.1056/NEJM199611073351902. PubMed PMID: 8875918.

3. Avierinos JF, Gersh BJ, Melton LJ, 3rd, et al. Natural history of asymptomatic mitral valve prolapse in the community. Circulation. 2002 Sep 10;106(11):1355-61. PubMed PMID: 12221052.

4. Enriquez-Sarano M, Avierinos JF, Messika-Zeitoun D, et al. Quantitative determinants of the outcome of asymptomatic mitral regurgitation. N Engl J Med. 2005 Mar 3;352(9):875-83. doi: 10.1056/NEJMoa041451. PubMed PMID: 15745978.

5. Coffey S, Cox B, Williams MJ. Lack of progress in valvular heart disease in the pre-transcatheter aortic valve replacement era: increasing deaths and minimal change in mortality rate over the past three decades. Am Heart J. 2014 Apr;167(4):562-567 e2. doi: 10.1016/j.ahj.2013.12.030. PubMed PMID:24655706. * This survey has highlighted important knowledge and skill gaps that may impede optimal care of the patient with MR.

6. Baumgartner H, Falk V, Bax JJ, et al. 2017 ESC/EACTS Guidelines for the management of valvular heart disease. Eur Heart J. 2017 Sep 21;38(36):2739-2791. doi: 10.1093/eurheartj/ehx391. PubMed PMID: 28886619.

7. Nishimura RA, Otto CM, Bonow RO, et al. 2017 AHA/ACC Focused Update of the 2014 AHA/ACC Guideline for the Management of Patients With Valvular Heart Disease: A Report of the American 
College of Cardiology/American Heart Association Task Force on Clinical Practice Guidelines. J Am Coll Cardiol. 2017 Jul 11;70(2):252-289. doi: 10.1016/j.jacc.2017.03.011. PubMed PMID: 28315732.

8. Mirabel M, lung B, Baron G, et al. What are the characteristics of patients with severe, symptomatic, mitral regurgitation who are denied surgery? Eur Heart J. 2007 Jun;28(11):1358-65. doi: 10.1093/eurheartj/ehm001. PubMed PMID: 17350971.

9. Bach DS, Awais M, Gurm HS, et al. Failure of guideline adherence for intervention in patients with severe mitral regurgitation. J Am Coll Cardiol. 2009 Aug 25;54(9):860-5. doi: 10.1016/j.jacc.2009.03.079. PubMed PMID: 19695468.

10. Jansen R, Kluin J, Chamuleau SA. Research versus clinical practice in asymptomatic patients with severe organic mitral regurgitation and preserved LV function. J Am Coll Cardiol. 2014 Oct 14;64(15):1639-40. doi: 10.1016/j.jacc.2014.07.964. PubMed PMID: 25301470.

11. lung B, Delgado V, Lazure $P$, et al. Educational needs and application of guidelines in the management of patients with mitral regurgitation. A European mixed-methods study. Eur Heart J. 2018 Apr 14;39(15):1295-1303. doi: 10.1093/eurheartj/ehx763. PubMed PMID: 29300869.

12. Dziadzko V, Clavel MA, Dziadzko M, et al. Outcome and undertreatment of mitral regurgitation: a community cohort study. Lancet. 2018 Mar 10;391(10124):960-969. doi: 10.1016/S01406736(18)30473-2. PubMed PMID: 29536860; PubMed Central PMCID: PMCPMC5907494. ** Even in a community with good access to treatment, isolated MR is substantially undertreated and associated with a high incidence of heart failure and excess mortality

13. El Sabbagh A, Reddy YNV, Nishimura RA. Mitral Valve Regurgitation in the Contemporary Era: Insights Into Diagnosis, Management, and Future Directions. JACC Cardiovasc Imaging. 2018 Apr;11(4):628-643. doi: 10.1016/j.jcmg.2018.01.009. PubMed PMID: 29622181.

14. Jung JC, Jang MJ, Hwang HY. Meta-Analysis Comparing Mitral Valve Repair Versus Replacement for Degenerative Mitral Regurgitation Across All Ages. Am J Cardiol. 2019 Feb 1;123(3):446-453. doi: 10.1016/j.amjcard.2018.10.024. PubMed PMID: 30471709.

15. Enriquez-Sarano M, Sundt TM, 3rd. Early surgery is recommended for mitral regurgitation. Circulation. 2010 Feb 16;121(6):804-11; discussion 812. doi:

10.1161/CIRCULATIONAHA.109.868083. PubMed PMID: 20159841.

16. Lazam S, Vanoverschelde JL, Tribouilloy C, et al. Twenty-Year Outcome After Mitral Repair Versus Replacement for Severe Degenerative Mitral Regurgitation: Analysis of a Large, Prospective, Multicenter, International Registry. Circulation. 2017 Jan 31;135(5):410-422. doi: 10.1161/CIRCULATIONAHA.116.023340. PubMed PMID: 27899396. * The very long-term data from the international MIDA registry is the strongest demonstration that MV repair should be preferred over MV replacement for patients with degenerative MR and a flail leaflet 
17. Badhwar V, Rankin JS, He X, et al. The Society of Thoracic Surgeons Mitral Repair/Replacement Composite Score: A Report of The Society of Thoracic Surgeons Quality Measurement Task Force. Ann Thorac Surg. 2016 Jun;101(6):2265-71. doi: 10.1016/j.athoracsur.2015.11.049. PubMed PMID: 26740032

18. Monin JL, Dehant $\mathrm{P}$, Roiron $\mathrm{C}$, et al. Functional assessment of mitral regurgitation by transthoracic echocardiography using standardized imaging planes diagnostic accuracy and outcome implications. J Am Coll Cardiol. 2005 Jul 19;46(2):302-9. doi: 10.1016/j.jacc.2005.03.064. PubMed PMID: 16022959. ** This study shows that a well-performed 2D-TTE allows to outline most of the mechanisms of MV disease, included the precise localization of prolapsed or flail segments.

19. Grewal J, Mankad S, Freeman WK, et al. Real-time three-dimensional transesophageal echocardiography in the intraoperative assessment of mitral valve disease. J Am Soc Echocardiogr. 2009 Jan;22(1):34-41. doi: 10.1016/j.echo.2008.11.008. PubMed PMID: 19131000.

20. Hien MD, Grossgasteiger M, Weymann A, et al. Reproducibility in echocardiographic two- and three-dimensional mitral valve assessment. Echocardiography. 2014 Mar;31(3):311-7. doi: 10.1111/echo.12365. PubMed PMID: 24028385.

21. Tsang W, Lang RM. Three-dimensional echocardiography is essential for intraoperative assessment of mitral regurgitation. Circulation. 2013 Aug 6;128(6):643-52; discussion 652. doi: 10.1161/CIRCULATIONAHA.112.120501. PubMed PMID: 23918185.

22. Suri RM, Vanoverschelde JL, Grigioni F, et al. Association between early surgical intervention vs watchful waiting and outcomes for mitral regurgitation due to flail mitral valve leaflets. JAMA. 2013 Aug 14;310(6):609-16. doi: 10.1001/jama.2013.8643. PubMed PMID: 23942679.

23. Zoghbi WA, Adams D, Bonow RO, et al. Recommendations for Noninvasive Evaluation of Native Valvular Regurgitation: A Report from the American Society of Echocardiography Developed in Collaboration with the Society for Cardiovascular Magnetic Resonance. J Am Soc Echocardiogr. 2017 Apr;30(4):303-371. doi: 10.1016/j.echo.2017.01.007. PubMed PMID: 28314623.

24. Enriquez-Sarano M, Akins CW, Vahanian A. Mitral regurgitation. Lancet. 2009 Apr 18;373(9672):1382-94. doi: 10.1016/S0140-6736(09)60692-9. PubMed PMID: 19356795.

25. Adams DH, Rosenhek R, Falk V. Degenerative mitral valve regurgitation: best practice revolution. Eur Heart J. 2010 Aug;31(16):1958-66. doi: 10.1093/eurheartj/ehq222. PubMed PMID: 20624767; PubMed Central PMCID: PMCPMC2921508.

26. Althunayyan A, Petersen SE, Lloyd G, et al. Mitral valve prolapse. Expert Rev Cardiovasc Ther. 2018 Nov 28. doi: 10.1080/14779072.2019.1553619. PubMed PMID: 30484338.

27. Chandra S, Salgo IS, Sugeng L, et al. Characterization of degenerative mitral valve disease using morphologic analysis of real-time three-dimensional echocardiographic images: objective insight 
into complexity and planning of mitral valve repair. Circ Cardiovasc Imaging. 2011 Jan;4(1):24-32. doi: 10.1161/CIRCIMAGING.109.924332. PubMed PMID: 20884831.

28. Sadeque SA, Barlow CW. Posterior leaflet mitral valve prolapse: One repair does not fit all. J Thorac Cardiovasc Surg. 2018 Nov;156(5):1867-1868. doi: 10.1016/j.jtcvs.2018.07.012. PubMed PMID: 30143374.

29. Lancellotti P, Moura L, Pierard LA, et al. European Association of Echocardiography recommendations for the assessment of valvular regurgitation. Part 2: mitral and tricuspid regurgitation (native valve disease). Eur J Echocardiogr. 2010 May;11(4):307-32. doi: 10.1093/ejechocard/jeq031. PubMed PMID: 20435783.

30. O'Gara PT, Grayburn PA, Badhwar V, et al. 2017 ACC Expert Consensus Decision Pathway on the Management of Mitral Regurgitation: A Report of the American College of Cardiology Task Force on Expert Consensus Decision Pathways. J Am Coll Cardiol. 2017 Nov 7;70(19):2421-2449. doi: 10.1016/j.jacc.2017.09.019. PubMed PMID: 29055505. ** This Expert Consensus Document proposes a structured approach to MV evaluation based on precise echocardiographic imaging which helps to clarify decision making.

31. De Bonis M, Alfieri O, Dalrymple-Hay M, et al. Mitral Valve Repair in Degenerative Mitral Regurgitation: State of the Art. Prog Cardiovasc Dis. 2017 Nov - Dec;60(3):386-393. doi: 10.1016/j.pcad.2017.10.006. PubMed PMID: 29117502.

32. Alreshidan M, Herron RD, Wei LM, et al. Surgical Techniques for Mitral Valve Repair: A Pathoanatomic Grading System. Semin Cardiothorac Vasc Anesth. 2019 Mar;23(1):20-25. doi: 10.1177/1089253218815465. PubMed PMID: 30516443; PubMed Central PMCID: PMCPMC6415492.

33. Varghese R, Itagaki S, Anyanwu AC, et al. Predicting systolic anterior motion after mitral valve reconstruction: using intraoperative transoesophageal echocardiography to identify those at greatest risk. Eur J Cardiothorac Surg. 2014 Jan;45(1):132-7; discussion 137-8. doi: 10.1093/ejcts/ezt234. PubMed PMID: 23657548.

34. La Canna G, Arendar I, Maisano F, et al. Real-time three-dimensional transesophageal echocardiography for assessment of mitral valve functional anatomy in patients with prolapserelated regurgitation. Am J Cardiol. 2011 May 1;107(9):1365-74. doi: 10.1016/j.amjcard.2010.12.048. PubMed PMID: 21371680. * This study shown that 3D-TEE provided more accurate mapping of $\mathrm{MV}$ prolapse than 2D imaging and 3D-TTE adding quantitative recognition of dominant and secondary lesions.

35. Mantovani F, Clavel MA, Vatury O, et al. Cleft-like indentations in myxomatous mitral valves by three-dimensional echocardiographic imaging. Heart. 2015 Jul;101(14):1111-7. doi:

10.1136/heartjnl-2014-307016. PubMed PMID: 25935768. 
36. Antoine C, Mantovani F, Benfari G, et al. Pathophysiology of Degenerative Mitral Regurgitation: New 3-Dimensional Imaging Insights. Circ Cardiovasc Imaging. 2018 Jan;11(1):e005971. doi: 10.1161/CIRCIMAGING.116.005971. PubMed PMID: 29321211.

37. Clavel MA, Mantovani F, Malouf J, et al. Dynamic phenotypes of degenerative myxomatous mitral valve disease: quantitative 3-dimensional echocardiographic study. Circ Cardiovasc Imaging. 2015 May;8(5). doi: 10.1161/CIRCIMAGING.114.002989. PubMed PMID: 25956922.

38. Genovese D, Addetia K, Kebed K, et al. First Clinical Experience With 3-Dimensional Echocardiographic Transillumination Rendering. JACC Cardiovasc Imaging. 2019 Feb 11. doi: 10.1016/j.jcmg.2018.12.012. PubMed PMID: 30772235.

39. McCarthy PM. When is your surgeon good enough? When do you need a "referent surgeon"? Curr Cardiol Rep. 2009 Mar;11(2):107-13. PubMed PMID: 19236826.

40. McCarthy PM. Three-dimensional echocardiography is not essential for intraoperative assessment of mitral regurgitation. Circulation. 2013 Aug 6;128(6):653-8; discussion 658. doi: 10.1161/CIRCULATIONAHA.112.120519. PubMed PMID: 23918186.

41. Koprivanac M, Kelava M, Alansari S, et al. Degenerative mitral valve disease-contemporary surgical approaches and repair techniques. Ann Cardiothorac Surg. 2017 Jan;6(1):38-46. doi:

10.21037/acs.2016.11.03. PubMed PMID: 28203540; PubMed Central PMCID: PMCPMC5293633.

42. Drake DH, Zimmerman KG, Hepner AM, et al. Echo-guided mitral repair. Circ Cardiovasc Imaging. 2014 Jan;7(1):132-41. doi: 10.1161/CIRCIMAGING.112.000458. PubMed PMID: 24218119.

** This monocentric study currently represents the strongest evidence that echocardiography can reliably identify repairable $\mathrm{MV}$ disease proving that echo-guided repair is associated with a higher rate of initial success than unguided historical and concomitant national controls.

43. Anyanwu AC, Itagaki S, Chikwe J, et al. A complexity scoring system for degenerative mitral valve repair. J Thorac Cardiovasc Surg. 2016 Jun;151(6):1661-70. doi: 10.1016/j.jtcvs.2016.01.033. PubMed PMID: 26936003. * This retrospective study proposed a complexity scale using a consensus approach, assigning weightings for the specific MV lesions derived from intraoperative observation.

44. Suri RM, Schaff HV, Dearani JA, et al. Survival advantage and improved durability of mitral repair for leaflet prolapse subsets in the current era. Ann Thorac Surg. 2006 Sep;82(3):819-26. doi: 10.1016/j.athoracsur.2006.03.091. PubMed PMID: 16928491.

45. Writing C, Nishimura RA, O'Gara PT, et al. 2019 AATS/ACC/ASE/SCAI/STS Expert Consensus Systems of Care Document: A Proposal to Optimize Care for Patients With Valvular Heart Disease: A Joint Report of the American Association for Thoracic Surgery, American College of Cardiology, American 
Society of Echocardiography, Society for Cardiovascular Angiography and Interventions, and Society of Thoracic Surgeons. 2019 Apr 3. doi: 10.1016/j.jacc.2018.10.007. PubMed PMID: 31010719.

46. Goldstone AB, Chiu P, Baiocchi M, et al. Mechanical or Biologic Prostheses for Aortic-Valve and Mitral-Valve Replacement. N Engl J Med. 2017 Nov 9;377(19):1847-1857. doi: 10.1056/NEJMoa1613792. PubMed PMID: 29117490.

47. Detaint $D$, Sundt TM, Nkomo VT, et al. Surgical correction of mitral regurgitation in the elderly: outcomes and recent improvements. Circulation. $2006 \mathrm{Jul}$ 25;114(4):265-72. doi: 10.1161/CIRCULATIONAHA.106.619239. PubMed PMID: 16847151.

48. Coutinho GF, Antunes MJ. Mitral valve repair for degenerative mitral valve disease: surgical approach, patient selection and long-term outcomes. Heart. 2017 Nov;103(21):1663-1669. doi: 10.1136/heartjnl-2016-311031. PubMed PMID: 28566474.

49. Coutinho GF, Correia PM, Branco C, et al. Long-term results of mitral valve surgery for degenerative anterior leaflet or bileaflet prolapse: analysis of negative factors for repair, early and late failures, and survival. Eur J Cardiothorac Surg. 2016 Jul;50(1):66-74. doi: 10.1093/ejcts/ezv470. PubMed PMID: 26792923.

50. Javadikasgari $\mathrm{H}$, Mihaljevic $\mathrm{T}$, Suri RM, et al. Simple versus complex degenerative mitral valve disease. J Thorac Cardiovasc Surg. 2018 Jul;156(1):122-129 e16. doi: 10.1016/j.jtcvs.2018.02.102. PubMed PMID: 29709354.

51. Gillinov M, Mick S, Suri RM. The Specialty of Mitral Valve Repair. J Am Coll Cardiol. 2017 Apr 24. doi: 10.1016/j.jacc.2017.01.059. PubMed PMID: 28476350.

52. Bonow RO, Adams DH. The Time Has Come to Define Centers of Excellence in Mitral Valve Repair. J Am Coll Cardiol. 2016 Feb 9;67(5):499-501. doi: 10.1016/j.jacc.2015.12.007. PubMed PMID: 26846947.

53. Chambers JB, Prendergast B, lung B, et al. Standards defining a 'Heart Valve Centre': ESC Working Group on Valvular Heart Disease and European Association for Cardiothoracic Surgery Viewpoint. Eur Heart J. 2017 Jul 21;38(28):2177-2183. doi: 10.1093/eurheartj/ehx370. PubMed PMID: 28838053.

54. Hannan EL, Samadashvili Z, Smith CR, et al. Mitral valve repair versus replacement for patients with preserved left ventricular function without heart failure symptoms. J Thorac Cardiovasc Surg. 2019 Apr;157(4):1432-1439 e2. doi: 10.1016/j.jtcvs.2018.08.091. PubMed PMID: 30482532.

55. Chikwe J, Toyoda N, Anyanwu AC, et al. Relation of Mitral Valve Surgery Volume to Repair Rate, Durability, and Survival. J Am Coll Cardiol. 2017 Apr 24. doi: 10.1016/j.jacc.2017.02.026. PubMed PMID: 28476349. 
56. Zamorano JL, Fernandez-Golfin C, Gonzalez-Gomez A. Quantification of mitral regurgitation by echocardiography. Heart. 2015 Jan;101(2):146-54. doi: 10.1136/heartjnl-2012-303498. PubMed PMID: 24780908.

57. Jansen R, Kluin J, Ray SG, et al. Identification of the Asymptomatic Patient With Severe Mitral Regurgitation: Discrepancy Between Research and Clinical Practice. Cardiol Rev. 2017 May/Jun;25(3):110-116. doi: 10.1097/CRD.0000000000000119. PubMed PMID: 27548683.

58. Seeburger J, Rinaldi M, Nielsen SL, et al. Off-pump transapical implantation of artificial neo-chordae to correct mitral regurgitation: the TACT Trial (Transapical Artificial Chordae Tendinae) proof of concept. J Am Coll Cardiol. 2014 Mar 11;63(9):914-9. doi: 10.1016/j.jacc.2013.07.090. PubMed PMID: 24076529.

59. Kiefer P, Meier S, Noack T, et al. Good 5-Year Durability of Transapical Beating Heart Off-Pump Mitral Valve Repair With Neochordae. Ann Thorac Surg. 2018 Aug;106(2):440-445. doi: 10.1016/j.athoracsur.2018.01.092. PubMed PMID: 29729267.

60. Colli A, Bagozzi L, Banchelli F, et al. Learning curve analysis of transapical NeoChord mitral valve repair. Eur J Cardiothorac Surg. 2018 Aug 1;54(2):273-280. doi: 10.1093/ejcts/ezy046. PubMed PMID: 29481644.

61. Colli A, Manzan E, Aidietis A, et al. An early European experience with transapical off-pump mitral valve repair with NeoChord implantation. Eur J Cardiothorac Surg. 2018 Sep 1;54(3):460-466. doi: 10.1093/ejcts/ezy064. PubMed PMID: 29514183. 


\section{Figures Legend}

Figure 1. Four imaging planes to assess the precise localization of prolapsed or flail segments. (A) Intercommissural plane assessing the continuity of the commissural areas. (B) Parasternal short-axis view showing the anterior leaflet $(A 1, A 2$, and $A 3)$ and the three scallops of the posterior leaflet $(P 1, P 2$, and $P 3)$. (C) Parasternal long-axis view showing the middle segments of anterior (A2) and posterior (P2) leaflets. (D) Apical four-chamber view showing the anterior para commissural zone (between P1 and P2). ANT. = anterior; $A O=$ descending aorta; $A V=$ aortic valve; $L A=$ left atrium; $L A A=$ left atrial appendage; $L V=$ left ventricle; POST. = posterior; $\mathrm{PV}=$ pulmonary vein; $\mathrm{RV}=$ right ventricle; $\mathrm{TV}=$ tricuspid valve. With permission from reference [18]

Figure 2. Depiction of mechanisms of MR as per the Carpentier classification. Type I leaflet motion is normal but can be associated with MR if there is annular dilation (secondary MR) or a leaflet perforation. Type II leaflet motion is excessive and is most commonly due to MV prolapse or flail leaflet. Type IIIb leaflet motion is restrictive in systole, commonly seen in the presence of left ventricular dilation (secondary MR), Type IIla leaflet motion is restrictive both in systole and diastole, commonly seen in rheumatic MV disease. With permission from reference.[23]

Figure 3. The schematically graduated protocol-driven echo guidance highlights that 3D-TEE is the essential moment to refine the pathoanatomy of the MV lesion(s) in order to implement access to high-quality surgical MV repair.

Figure 4. Pre-repair echocardiographic measurements in a at risk of SAM after MV repair. The mechanism of post-repair SAM involves anatomic and hemodynamic factors. Essentially, SAM is explained by the presence of excess leaflet tissue relative to annular size creating a larger surface area on which drag forces act. Although both excessive anterior and posterior leaflet tissue predispose to SAM, a long posterior relative to anterior leaflet length, in which the coaptation line is displaced anteriorly into the left ventricular outflow tract, is a particular risk factor.

Figure 5. Volume rendering image of 3D zoom data set of $M V$ for quantitative evaluation of leaflet tip displacement above anulus hinge point along intercommissural coaptation line. Coesistence of A2 secondary lesion with P2 main prolapse, elicited with 3D-TEE cropping. Video 4.

Figure 6. Retrospective modeling of data from 668 consecutive patients who underwent surgery for mitral valve prolapse. Mitral valve repair was successfully performed in 667 patients (repair rate: 99.9\%). A 
complexity scoring scale was developed using a consensus approach, assigning a score to each valve. Adapted with permission from reference[43]

\section{Table Legend}

Table 1. Mitral valve surgical repair techniques in degenerative mitral regurgitation due to leaflet prolapse according to the echocardiographic pathoanatomic features and corresponding operative findings. Abbreviation: PTFE, polytetrafluoroethylene. Adapted with permission from reference [32]

\section{Video Legend}

Video 1. En face view of the MV on 3D-TEE showing the multiple mechanisms causing MR with asymmetric pattern. The image depicts myxomatous degeneration with billowing of the anterior leaflet and the failure of coaptation of leaflets in the P2 and P3 regions. These segments are restricted to closure in systole.

Video 2. Example of Barlow's disease with multi-segment prolapse and excess leaflet tissue. With 3D-TEE imaging, predominant involvement of P2 is identified.

Video 3. En face view of the MV on 3D-TEE demonstrating Barlow's disease with bileaflet, multisegmental prolapse and predominant involvement of A3. Deep indentations of the posterior leaflet can also be appreciated.

Video 4. Surgical view of MV showing dominant prolapse and flail of middle scallop of posterior leaflet and possible secondary prolapse of medial segments of both leaflets. Cross-section reference of cutting plane to guide prolapse measurement.

Video 5. Predominant medial commissural prolapse lesion.

Video 6. Predominant lateral commissural prolapse lesion.

Video 7. Posterior leaflet cleft involving P1/P2. The cleft is in the region of the scallop that normally separates P1 and P2 and therefore may be considered a "deep scallop" which extends from the free edge to the annulus.

Video 8. Patient at risk of SAM after MV repair, best seen in the midesophageal long-axis view 2D-TEE at end-systole. 
Figure 1

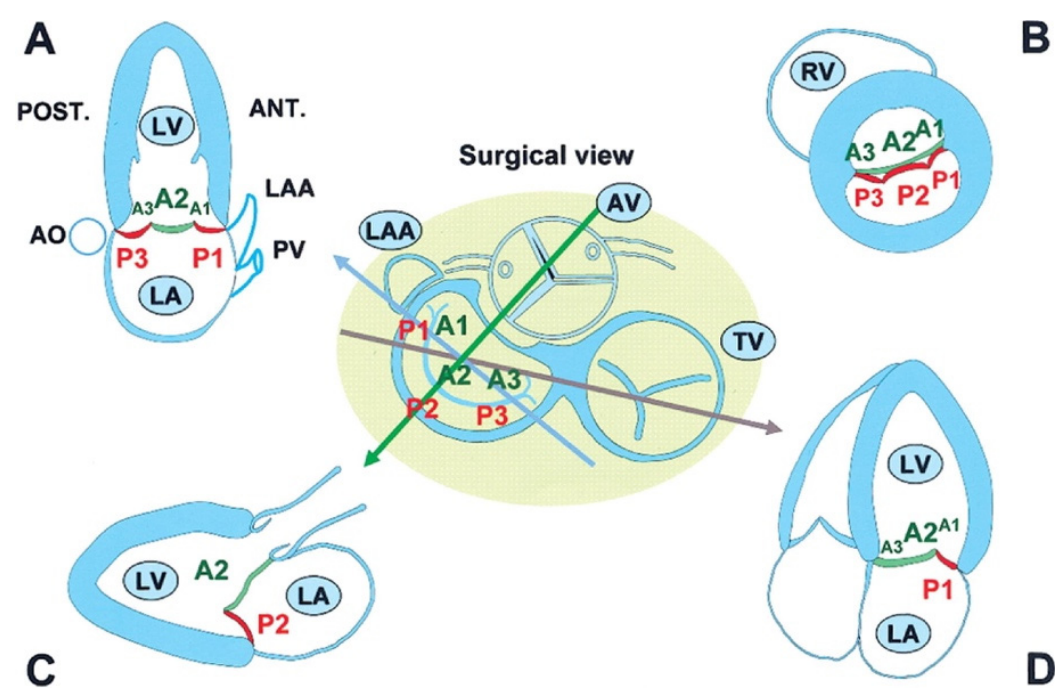

Figure 2

\begin{tabular}{|c|c|c|c|l|l|}
\hline \multicolumn{2}{|c|}{$\begin{array}{c}\text { Type I } \\
\text { Normal Leaflet } \\
\text { Motion }\end{array}$} & \multicolumn{2}{c|}{$\begin{array}{c}\text { Type II } \\
\text { Excessive Leaflet } \\
\text { Motion }\end{array}$} & \multicolumn{2}{c|}{$\begin{array}{c}\text { Type III } \\
\text { Restricted Leaflet } \\
\text { Motion }\end{array}$} \\
\hline $\begin{array}{c}\text { Annular } \\
\text { Dilation }\end{array}$ & Perforation & Prolapse & Flail & $\begin{array}{l}\text { a } \\
\text { Thickening/ }\end{array}$ & Fusion \\
Dilation
\end{tabular}

Figure 3 


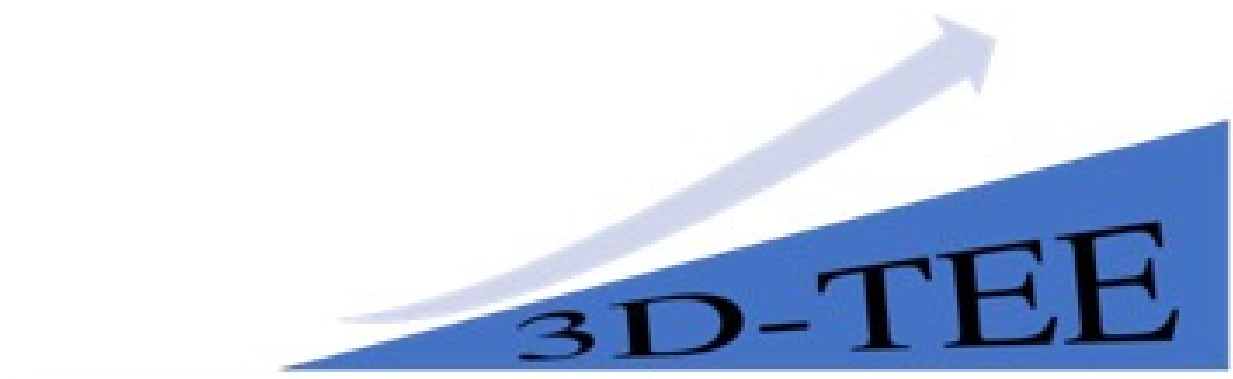

\begin{tabular}{l|l}
$\begin{array}{l}\text { Focus on the } \\
\text { principal lesion }\end{array}$ & $\begin{array}{l}\text { Quantify the } \\
\text { principal lesion }\end{array}$ \\
\hline
\end{tabular}

\section{Identify potential secondary mechanisms}

that could be masked by the principal lesion

Figure 4

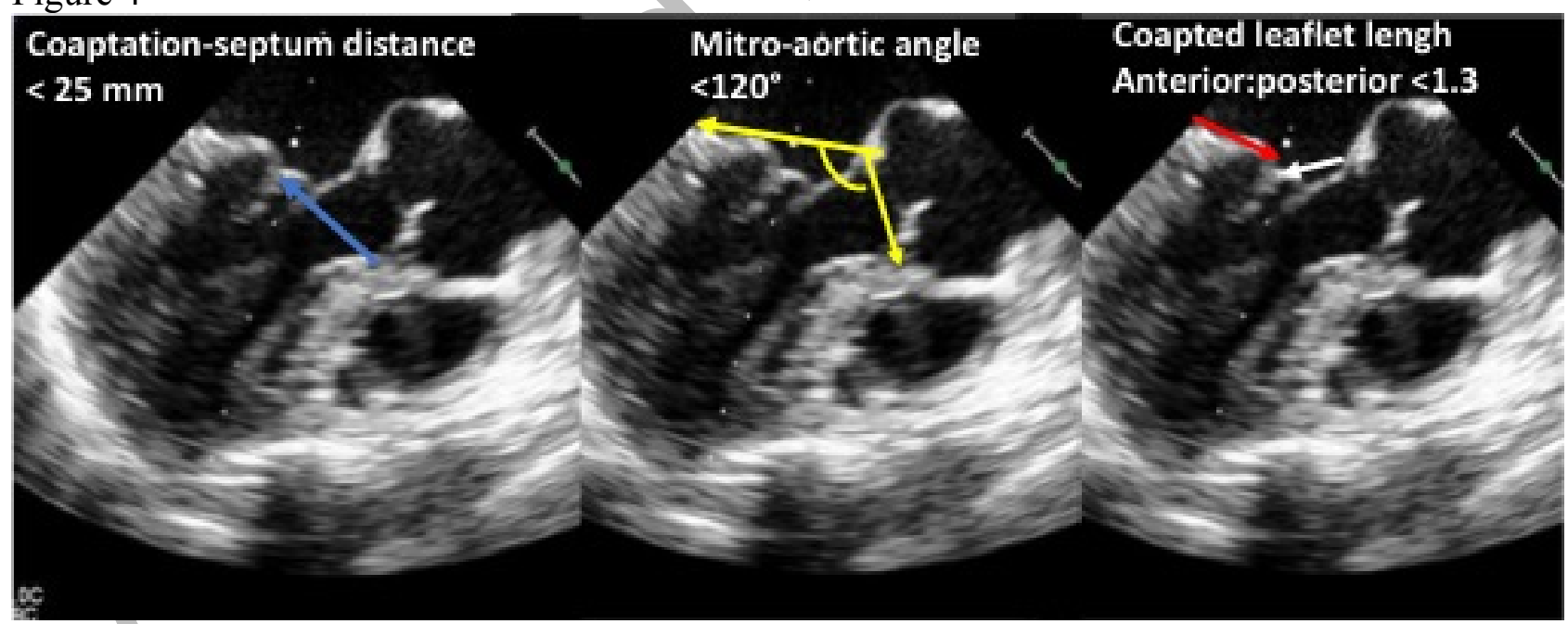

Figure 5 


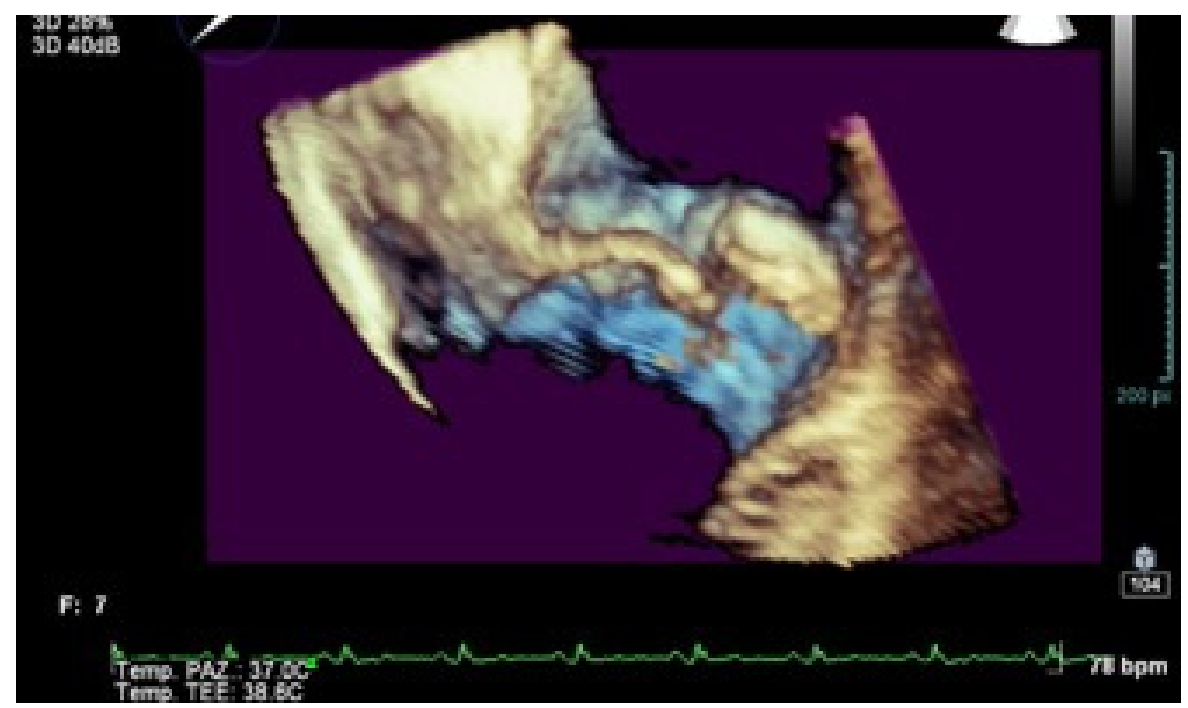

Figure 6

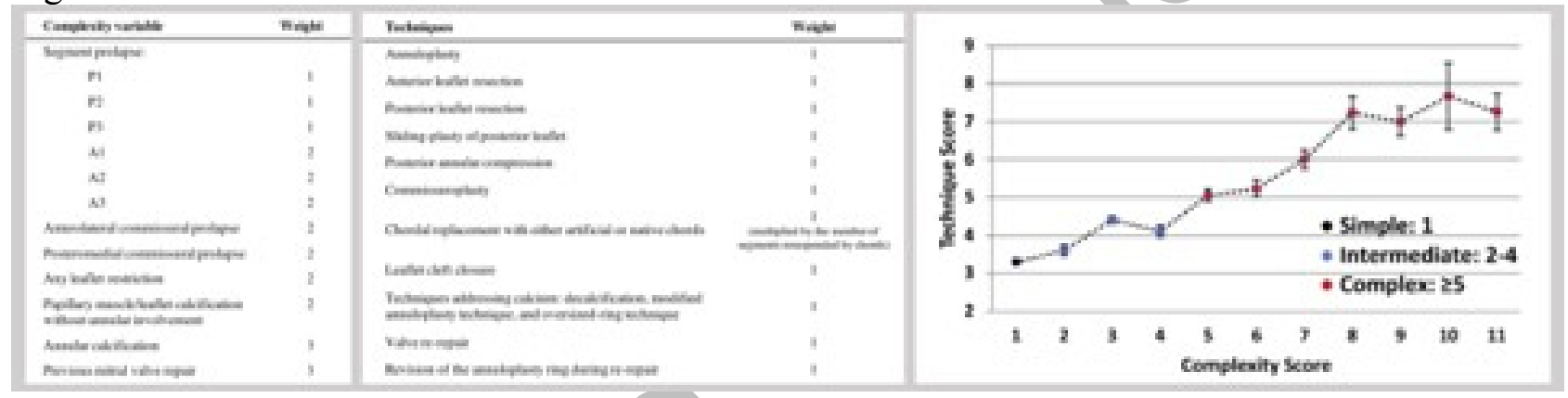

\begin{tabular}{|c|c|c|c|c|}
\hline GRADE & PATHOANATOMIC FEATURES & ECHOCARDIOGRAPHY & OPERATIVE FINDINGS & REPAIR OPTIONS \\
\hline 1 & $\begin{array}{l}\text { - Annular dilatation } \\
\text { - Isolated posterior leaflet prolapse } \\
\text { or single segment flail }\end{array}$ & & & $\begin{array}{l}\text { - Focal resection and } \\
\text { valvuloplasty with ring } \\
\text { annuloplasty } \\
\text { - PTFE neochord support } \\
\text { - Ring/band annuloplasty }\end{array}$ \\
\hline 2 & $\begin{array}{l}\text { - Diffuse myxomatous disease } \\
\text { predominantly of posterior leaflet } \\
\text { (forme fruste) }\end{array}$ & & & $\begin{array}{l}\text { - Partial resection and sliding } \\
\text { leaflet valvuloplasty } \\
\text { - Multi-segment PTFE neochord } \\
\text { support } \\
\text { - Ring/band annuloplasty }\end{array}$ \\
\hline 3 & $\begin{array}{l}\text { - Diffuse bi-leaflet myxomatous } \\
\text { disease (Barlow's) } \\
\text { - Anterior leaflet flail } \\
\text { - Multi-segment flail } \\
\text { - Focal posterior mitral annular } \\
\text { calcification }\end{array}$ & & & $\begin{array}{l}\text { - Partial resection and sliding } \\
\text { leaflet valvuloplasty } \\
\text { - Multi-segment PTFE neochord } \\
\text { support } \\
\text { - Secondary chordal transfer } \\
\text { - Focal calcium resection } \\
\text { - Ring/band annuloplasty }\end{array}$ \\
\hline 4 & $\begin{array}{l}\text { - Endocarditis } \pm \text { leaflet perforation } \\
\text { or annular abscess } \\
\text { - Rheumatic Type IIIA disease } \\
\text { - Secondary Type IIIB disease with } \\
\text { severe tethering } \\
\text { - Severe mitral annular calcification }\end{array}$ & & & $\begin{array}{l}\text { - Reconstruction with patch } \\
\text { augmentation } \\
\text { - Subvalvular mobilization } \\
\text { - Radical annular reconstruction } \\
\text { - Ring/band annuloplasty }\end{array}$ \\
\hline
\end{tabular}

